\title{
A Questionnaire-Based Survey on the Impact of the COVID-19 Pandemic on Gastrointestinal Endoscopy in Asia
}

\author{
Koji Otani ${ }^{a}$ Toshio Watanabe ${ }^{b}$ Akira Higashimori ${ }^{a}$ Hidekazu Suzuki ${ }^{c}$ Takeshi Kamiya $^{d}$ \\ Akiko Shiotani $^{\mathrm{e}}$ Mitsushige Sugimoto $^{\mathrm{f}}$ Akihito Nagaharag Shin Fukudo ${ }^{\mathrm{h}}$ Satoshi Motoya ${ }^{\mathrm{i}}$ \\ Satoru Yamaguchi ${ }^{j}$ Qi Zhu ${ }^{k}$ Francis K.L. Chan' Ki-Baik Hahm ${ }^{m}$ Maria Carla Tablante ${ }^{\text {n }}$ \\ Varayu Prachayakul ${ }^{\circ}$ Murdani Abdullah $^{p}$ Tiing Leong Ang ${ }^{q} \quad$ Kazunari Murakami $^{r}$ \\ The International Gastrointestinal Consensus Symposium Study Group
}

${ }^{a}$ Department of Gastroenterology, Osaka City University Graduate School of Medicine, Osaka, Japan; ${ }^{b}$ Department of Premier Preventive Medicine, Osaka City University Graduate School of Medicine, Osaka, Japan; 'Divisions of Gastroenterology and Hepatology, Department of Internal Medicine, Tokai University School of Medicine, Isehara, Japan; ${ }^{d}$ Department of Medical Innovation, Nagoya City University Graduate School of Medical Sciences, Nagoya, Japan; 'Division of Gastroenterology, Department of Internal Medicine, Kawasaki Medical School, Okayama, Japan; fDepartment of Gastroenterological Endoscopy, Tokyo Medical University, Tokyo, Japan; ${ }^{9}$ Department of Gastroenterology, Juntendo University School of Medicine, Tokyo, Japan; hepartment of Behavioral Medicine, Tohoku University Graduate School of Medicine, Sendai, Japan; 'IBD Center, Hokkaido Prefectural Welfare Federation of Agricultural Cooperative, Sapporo-Kosei General Hospital, Sapporo, Japan; jDepartment of Surgical Oncology, Dokkyo Medical University, Mibu, Japan; ksinoUnited Health, Ruijin Hospital, Shanghai Jiaotong University School of Medicine, Shanghai, China; 'Department Medicine and Therapeutics, Institute of Digestive Disease, Faculty of Medicine, The Chinese University of Hong Kong, Shatin, Hong Kong SAR; mDigestive Disease Center, CHA University School of Medicine and CHA University Bundang Medical Center, Seongnam, South Korea; nDepartment Internal Medicine, Section of Gastroenterology and Hepatology, University of Santo Tomas Hospital, Manila, Philippines; ${ }^{\circ}$ Division of Gastroenterology, Department Internal Medicine, Faculty of Medicine, Siriraj

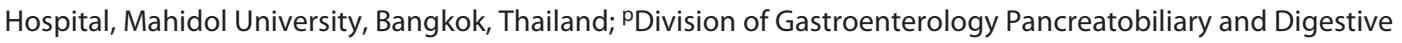
Endoscopy, Department of Internal Medicine, Dr. Cipto Mangunkusumo Hospital, Faculty of Medicine, Universitas Indonesia, Jakarta, Indonesia; ${ }^{\circ D e p a r t m e n t ~ o f ~ G a s t r o e n t e r o l o g y ~ a n d ~ H e p a t o l o g y, ~ C h a n g i ~ G e n e r a l ~ H o s p i t a l, ~ D u k e-~}$ NUS Medical School, Yong Loo Lin School of Medicine, National University of Singapore, Singapore, Singapore; 'Department of Gastroenterology, Faculty of Medicine, Oita University, Oita, Japan

\section{Keywords}

COVID-19. Severe acute respiratory syndrome coronavirus 2 - Endoscopy Personal protective equipment $\cdot$ Asia

\begin{abstract}
Introduction: The COVID-19 outbreak abruptly restricted gastrointestinal (GI) endoscopy services during the first wave of the pandemic. We aimed to assess the impact of COVID-19 on the practice of Gl endoscopy in Asian countries. Methods: This was an International Questionnaire-based Internet Survey conducted at multiple facilities by the International Gastrointestinal Consensus Symposium. A total of 166
\end{abstract}

Correspondence to:

ToshioWatanabe, watanabet@med.osaka-cu.ac.jp 
respondents in Japan, China, Hong Kong, South Korea, Philippines, Thailand, Indonesia, and Singapore participated in this study. Results: The volume of endoscopic screening or follow-up endoscopies and therapeutic endoscopies were markedly reduced during the first wave of the pandemic, which was mainly attributed to the decreased number of outpatients, cancellations by patients, and adherence to the guidelines of academic societies. The most common indications for $\mathrm{Gl}$ endoscopy during the first wave were $\mathrm{Gl}$ bleeding, cholangitis or obstructive jaundice, and a highly suspicious case of neoplasia. The most common Gl symptoms of COVID-19 patients during the infected period included diarrhea, nausea, and vomiting. The pandemic exacerbated some $\mathrm{Gl}$ diseases, such as functional dyspepsia and irritable bowel syndrome. There were cases with delayed diagnosis of cancers due to postponed endoscopic procedures, and the prescription of proton pump inhibitors/potassium-competitive acid blockers, steroids, immunosuppressive agents, and biologics was delayed or canceled. The personal protective equipment used during endoscopic procedures for high-risk patients were disposable gloves, disposable gowns, N95 or equivalent masks, and face shields. However, the devices on the patient side during endoscopic procedures included modified surgical masks, mouthpieces with filters, and disposable vinyl boxes or aerosol boxes covering the head. Furthermore, the time for education, basic research, clinical research, and daily clinical practice decreased during the first wave. Conclusion: This study demonstrated that the COVID-19 pandemic profoundly affected the method of performing Gl endoscopy and medical treatment for patients with $\mathrm{Gl}$ diseases in Asian countries.

(c) 2021 S. Karger AG, Basel

\section{Introduction}

COVID-19, caused by severe acute respiratory syndrome coronavirus 2 (SARS-CoV-2), emerged and spread in December 2019 [1]. The outbreak rapidly spread worldwide and was declared a global pandemic by the World Health Organization on March 11, 2020. During the first wave of the pandemic, governments of many countries worldwide imposed immigration restrictions, quarantine, contact tracing, and several behavioral restrictions, such as lockdown of cities, social distancing, and closure of school and commercial facilities. These measures have largely changed our daily lifestyle [2-4].

The major transmission routes of SARS-CoV-2 are droplets, aerosols, or contact infections, and the possibility of transmission through the fecal-oral route has also been suggested [5-9]. Medical staff working in the endoscopic units are potentially at high risk for SARS-CoV-2 infection because the transmission occurs by inhalation of droplets or aerosols generated by coughing or the vomiting reflex of the examinees during endoscopic procedures [10]. Consequently, academic societies of gastrointestinal (GI) endoscopy, such as the Asian Pacific Society for Digestive Endoscopy (APSDE) and Japan Gastroenterological Endoscopy Society, issued statements on triaging endoscopic procedures, setting of endoscopic units including the number of medical staff, and appropriately using personal protective equipment (PPE) to protect patients and medical staff during the pandemic $[11,12]$. Nonurgent screening or follow-up upper GI endoscopies tended to be postponed, especially during the first wave. Medical staff and endoscopic booths were limited to maintain the appropriate distance from each other. Although the PPE protocols during endoscopic procedures against SARS-CoV-2 infection varied according to the country and institution, it was recommended by academic societies that the standard precautions should be followed for all patients during GI endoscopies. Furthermore, most medical staff used goggles, masks, gloves, hairnets, and long-sleeved gowns. However, serious problems appeared owing to the new infection control policy during GI endoscopy. Incidentally, there were cases in which the detection of GI cancers was delayed because of postponed endoscopy [13]. Furthermore, in some institutions and countries, the lack of adequate supply of PPE restricted the practice of GI endoscopy and prohibited residents and fellows from participating in endoscopic training to preserve PPE and minimize exposure [14].

Although GI endoscopy services were abruptly restricted during the first wave of the pandemic, they gradually became available on a regular schedule after a while. However, resuming full endoscopy requires accurate understanding of the actual condition of GI endoscopy, performing risk assessment before endoscopy, setting up of

Fig. 1. Impact of the COVID-19 pandemic on the number of GI endoscopies. a The degree of the decrease in the number of screening or follow-up endoscopies compared to before the pandemic during and after the first wave. $\mathbf{b}$ The degree of the decrease in the number of therapeutic endoscopies compared to before the pandemic during and after the first wave. $\mathbf{c}$ The degree of the decrease in the number of urgent endoscopies (on call) compared to before the pandemic during and after the first wave. $\mathbf{d}$ The reason for the decrease in GI endoscopic procedures during the first wave. e The reason for the decrease in GI endoscopic procedures after the first wave. PPE, personal protective equipment; GI, gastrointestinal.

(For figure see next page.) 

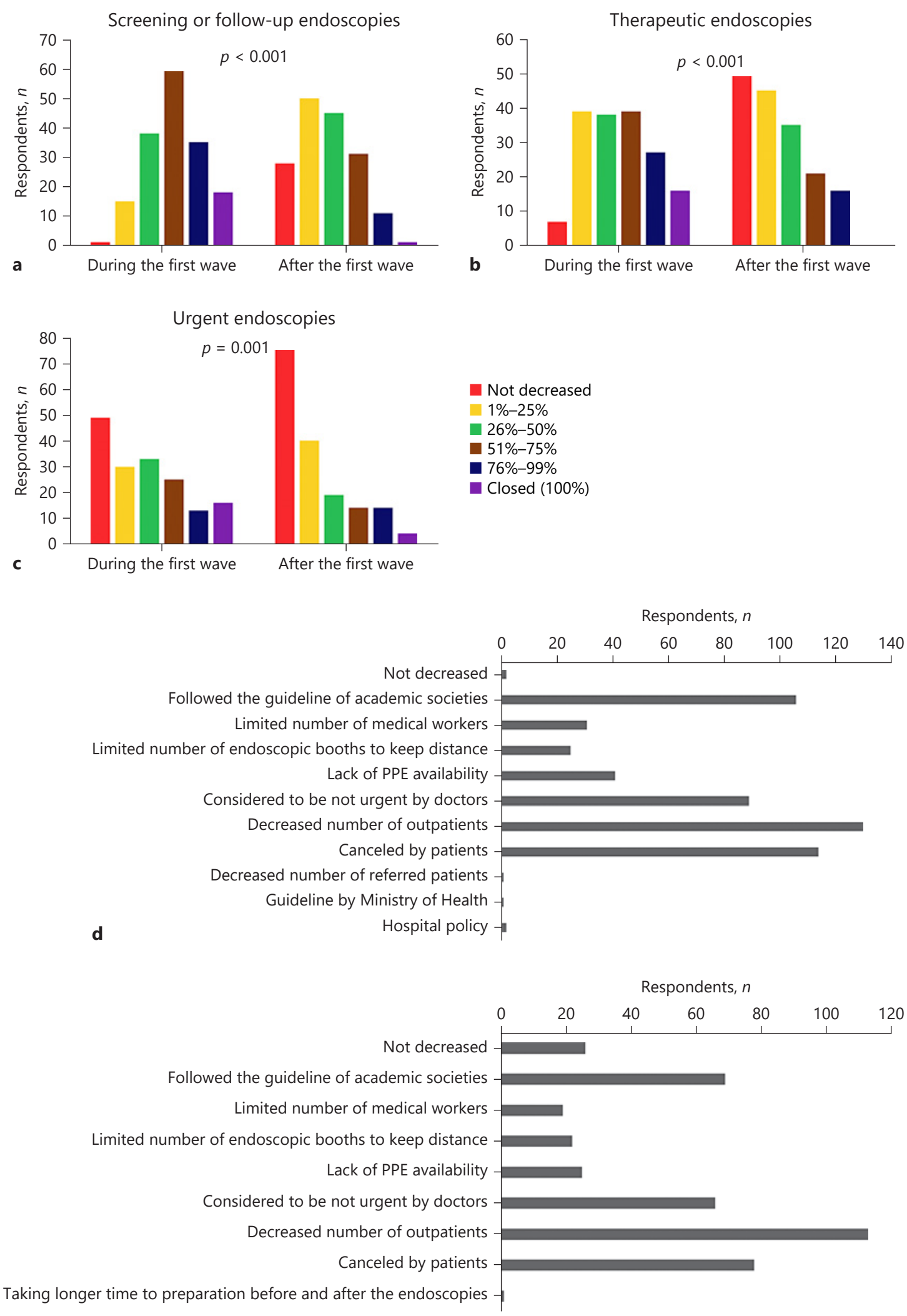
endoscopic units, being aware of changes in the medical treatment for GI diseases, and understanding the use of PPE. Furthermore, the impact of the COVID-19 pandemic on education, research, and clinical practice has not been quantified. Given the above circumstances and considering that the COVID-19 outbreak first occurred in Asia, we conducted a questionnaire-based survey to assess the impact of COVID-19 on the practice of GI endoscopy at multiple facilities in East and Southeast Asian countries.

\section{Methods}

\section{Study Design}

This was an International Questionnaire-based Internet Survey conducted at multiple facilities by the International Gastrointestinal Consensus Symposium (IGICS).

\section{Study Participants}

The questionnaire, consisting of 33 questions related to the impact of COVID-19 on GI endoscopy practice, was distributed to a total of 166 participants belonging to several institutions including universities, public and private hospitals, medical checkup institutions, and private clinics in Japan, China, Hong Kong, South Korea, Philippines, Thailand, Indonesia, and Singapore via the Internet survey by IGICS. The survey started at the beginning of October 2020, and the responses were collected by the end of November 2020. The questionnaire focused on the following: demographics, endoscopy, medical treatment for COVID-19, PPE, and others. The details of the questionnaire are described in online supplementary Table 1 (see www.karger.com/doi/10.1159/000520287 for all online suppl. material).

\section{Statistical Analysis}

Data are expressed as the number of respondents and the percentage of responses of the 166 participants. Questionnaire items indicated to select one answer for the difference between during the first wave and after the first wave were compared using a Pearson's $\chi^{2}$ test or Fisher's exact test. Statistical calculations and analyses were performed using IBM SPSS Statistics Version 26 (IBM Corporation, Armonk, NY, USA). The statistical significance was set at $p$ values $<0.05$.

\section{Results}

\section{Background of Respondents in Asian Countries}

The questionnaire was distributed to 166 participants and all completed the questionnaire (response rate $=$ $100.0 \%)$. Of the participants, 60 were from Japan (36.1\%), 26 from China (15.7\%), 15 from Hong Kong (9.0\%), 7 from South Korea (4.2\%), 23 from Philippines (13.9\%), 8 from Thailand (4.8\%), 18 from Indonesia (10.8\%), and 9 from Singapore (5.4\%). The specialty of the respondents was mostly gastroenterology $(88.6 \%)$, followed by internal medicine $(9.0 \%)$, and surgery (1.8\%). Most of the respondents belonged to university hospitals (41.0\%), followed by private hospitals (28.3\%), and public hospitals (27.1\%). Their clinical roles were mostly staff $(48.8 \%)$, followed by directors (30.1\%), and fellows (10.2\%). The data are listed in online supplementary Table 2.

\section{Impact of the COVID-19 Pandemic on the Practice of GI Endoscopy}

The number of GI endoscopic procedures for screening or follow-up endoscopies and therapeutic endoscopies decreased by $51-75 \%$ in the highest number of answers during the first wave of the pandemic (59 [35.5\%] and 39 [23.5\%] of respondents, respectively), compared to before the pandemic. Although the number of these endoscopic procedures did not completely return to what it was before the pandemic, it significantly increased after the first wave, compared to during the first wave (Fig. 1a, b). In particular, the number of screening or follow-up endoscopies and therapeutic endoscopies did not decrease much after the first wave in Thailand (5 [62.5\%] and $6[75.0 \%]$ of respondents, respectively), compared to before the pandemic (Table 1). In contrast, the decrease in the number of urgent GI endoscopic procedures was less, and it did not decrease in 49 (29.5\%) of the respondents during the first wave (Fig. 1c). The number of urgent endoscopies did not decrease during the first wave and after the first wave (10 [66.7\%] and 12 [80.0\%] of respondents, respectively), particularly in Hong Kong (Table 1). The most common reason for the decrease in GI endoscopic procedures during the first wave was decreased number of outpatients (130 [78.3\%]), followed by cancellations by patients (114 [68.7\%]), and adherence to the guidelines of academic societies (106 [63.9\%]). Notably, 31 (18.7\%) and 41 (24.7\%) respondents answered that a limited number of medical workers and lack of PPE availability were causes for the decrease, respectively (Fig. 1d). Even after the first wave of the pandemic, decreased number of outpatients (113 [68.1\%]), cancellations by patients $(78[47.0 \%])$, and adherence to the guidelines of academic societies (69 [41.6\%]) were the common reasons for the decrease in GI endoscopic procedures (Fig. 1e).

The most common indication for endoscopy during the first wave was GI bleeding or suspected GI bleeding (140 [84.3\%]), followed by cholangitis or obstructive jaundice (117 [70.5\%]), and highly suspicious cases of neoplasia (103 [62.0\%]). Although the number of institutions where GI endoscopy was performed without re- 
Table 1. The decrease in the number of Gl endoscopies and PPE used during endoscopic procedures by country in Asia

\begin{tabular}{|c|c|c|c|c|c|c|c|c|}
\hline \multicolumn{9}{|c|}{ The degree of the decrease in the number of screening or follow-up endoscopies compared to before pandemic during the first wave, $n$ (\%) } \\
\hline Not decreased & $1(1.7)$ & $0(0.0)$ & $0(0.0)$ & $0(0.0)$ & $0(0.0)$ & $0(0.0)$ & $0(0.0)$ & $0(0.0)$ \\
\hline $26-50 \%$ & $14(23.3)$ & $5(19.2)$ & $3(20.0)$ & 5 (71.4) & $0(0.0)$ & $3(37.5)$ & $5(27.8)$ & $3(33.3)$ \\
\hline $51-75 \%$ & 22 (36.7) & $6(23.1)$ & $5(33.3)$ & 1 (14.3) & 11 (47.8) & $1(12.5)$ & $9(50.0)$ & 4 (44.4) \\
\hline $76-99 \%$ & $9(15.0)$ & $8(30.8)$ & $6(40.0)$ & 1 (14.3) & $8(34.8)$ & $2(25.0)$ & $1(5.6)$ & $0(0.0)$ \\
\hline \multicolumn{9}{|c|}{ The degree of the decrease in the number of screening or follow-up endoscopies compared to before pandemic after the first wave, $n$ (\%) } \\
\hline Not decreased & $11(18.3)$ & $8(30.8)$ & $1(6.7)$ & $0(0.0)$ & $0(0.0)$ & $5(62.5)$ & $0(0.0)$ & $3(33.3)$ \\
\hline $1-25 \%$ & $22(36.7)$ & $7(26.9)$ & $4(26.7)$ & $5(71.4)$ & $2(8.7)$ & $2(25.0)$ & $5(27.8)$ & $3(33.3)$ \\
\hline $26-50 \%$ & 11 (18.3) & 9 (34.6) & $6(40.0)$ & $0(0.0)$ & $11(47.8)$ & $0(0.0)$ & $6(33.3)$ & $2(22.2)$ \\
\hline $51-75 \%$ & $7(11.7)$ & $2(7.7)$ & $4(26.7)$ & $1(14.3)$ & $9(39.1)$ & $1(12.5)$ & $6(33.3)$ & $1(11.1)$ \\
\hline $76-99 \%$ & $8(13.3)$ & $0(0.0)$ & $0(0.0)$ & 1 (14.3) & $1(4.3)$ & $0(0.0)$ & $1(5.6)$ & $0(0.0)$ \\
\hline $26-50 \%$ & 16 (26.7) & 5 (19.2) & $3(20.0)$ & $2(28.6)$ & $3(13.0)$ & $2(25.0)$ & $6(33.3)$ & $1(11.1)$ \\
\hline $51-75 \%$ & 10 (16.7) & 5 (19.2) & $3(20.0)$ & 1 (14.3) & $13(56.5)$ & 1 (12.5) & $4(22.2)$ & $2(22.2)$ \\
\hline $76-99 \%$ & $9(15.0)$ & 7 (26.9) & $4(26.7)$ & $0(0.0)$ & $3(13.0)$ & $1(12.5)$ & $3(16.7)$ & $0(0.0)$ \\
\hline Closed (100) & $4(6.7)$ & $8(30.8)$ & $0(0.0)$ & $1(14.3)$ & $2(8.7)$ & $1(12.5)$ & $0(0.0)$ & $0(0.0)$ \\
\hline \multicolumn{9}{|c|}{ The degree of the decrease in the number of therapeutic endoscopies compared to before pandemic after the first wave, $n$ (\%) } \\
\hline Not decreased & $23(38.3)$ & $8(30.8)$ & $3(20.0)$ & $1(14.3)$ & $1(4.3)$ & $6(75.0)$ & $2(11.1)$ & $5(55.6)$ \\
\hline $1-25 \%$ & $17(28.3)$ & $6(23.1)$ & $7(46.7)$ & $4(57.1)$ & $3(13.0)$ & $2(25.0)$ & $5(27.8)$ & 1 (11.1) \\
\hline $26-50 \%$ & $6(10.0)$ & $8(30.8)$ & $3(20.0)$ & $1(14.3)$ & $9(39.1)$ & $0(0.0)$ & $6(33.3)$ & $2(22.2)$ \\
\hline $51-75 \%$ & $5(8.3)$ & $4(15.4)$ & $1(6.7)$ & $0(0.0)$ & $8(34.8)$ & $0(0.0)$ & $3(16.7)$ & $0(0.0)$ \\
\hline $76-99 \%$ & $9(15.0)$ & $0(0.0)$ & $1(6.7)$ & $1(14.3)$ & $2(8.7)$ & $0(0.0)$ & $2(11.1)$ & $1(11.1)$ \\
\hline Closed (100) & $0(0.0)$ & $0(0.0)$ & $0(0.0)$ & $0(0.0)$ & $0(0.0)$ & $0(0.0)$ & $0(0.0)$ & $0(0.0)$ \\
\hline \multicolumn{9}{|c|}{ The degree of the decrease in the number of urgent endoscopies (on call) compared to before pandemic during the first wave, $n$ (\%) } \\
\hline $1-25 \%$ & $14(23.3)$ & $7(26.9)$ & $0(0.0)$ & $4(57.1)$ & $6(26.1)$ & $2(25.0)$ & $6(33.3)$ & $1(11.1)$ \\
\hline $26-50 \%$ & $4(6.7)$ & $2(7.7)$ & $1(6.7)$ & $0(0.0)$ & $6(26.1)$ & $0(0.0)$ & $6(33.3)$ & $0(0.0)$ \\
\hline $51-75 \%$ & $3(5.0)$ & $0(0.0)$ & $0(0.0)$ & $0(0.0)$ & $7(30.4)$ & $0(0.0)$ & $3(16.7)$ & $1(11.1)$ \\
\hline $76-99 \%$ & $10(16.7)$ & $0(0.0)$ & $1(6.7)$ & $0(0.0)$ & $2(8.7)$ & $0(0.0)$ & $0(0.0)$ & $1(11.1)$ \\
\hline Closed (100) & $1(1.7)$ & $0(0.0)$ & $1(6.7)$ & $1(14.3)$ & $0(0.0)$ & $0(0.0)$ & $1(5.6)$ & $0(0.0)$ \\
\hline \multicolumn{9}{|c|}{ PPE in short supply during the first wave, $n(\%)$} \\
\hline None & $5(8.3)$ & $8(30.8)$ & $1(6.7)$ & $2(28.6)$ & $4(17.4)$ & $0(0.0)$ & $4(22.2)$ & 7 (77.8) \\
\hline Face shield & $34(56.7)$ & $10(38.5)$ & $8(53.3)$ & $4(57.1)$ & $7(30.4)$ & $6(75.0)$ & $12(66.7)$ & $0(0.0)$ \\
\hline Goggle & $30(50.0)$ & $9(34.6)$ & $6(40.0)$ & $3(42.9)$ & $8(34.8)$ & $6(75.0)$ & $8(44.4)$ & $0(0.0)$ \\
\hline Surgical mask & 46 (76.7) & $12(46.2)$ & $9(60.0)$ & $3(42.9)$ & $9(39.1)$ & $8(100.0)$ & $9(50.0)$ & $1(11.1)$ \\
\hline N95, FFP 2, or DS2 & $41(68.3)$ & $17(65.4)$ & $13(86.7)$ & $3(42.9)$ & 19 (82.6) & $7(87.5)$ & $14(77.8)$ & $2(22.2)$ \\
\hline Disposable gloves & $31(51.7)$ & $5(19.2)$ & $3(20.0)$ & $3(42.9)$ & $3(13.0)$ & $4(50.0)$ & $12(66.7)$ & $0(0.0)$ \\
\hline Hairnet & $25(41.7)$ & $3(11.5)$ & $1(6.7)$ & $1(14.3)$ & $2(8.7)$ & $4(50.0)$ & $12(66.7)$ & $0(0.0)$ \\
\hline Disposable gown & $50(83.3)$ & 7 (26.9) & $12(80.0)$ & 3 (42.9) & $15(65.2)$ & $6(75.0)$ & $12(66.7)$ & $1(11.1)$ \\
\hline Shoe covers & $15(25.0)$ & $4(15.4)$ & $0(0.0)$ & $2(28.6)$ & $6(26.1)$ & $5(62.5)$ & $10(55.6)$ & $0(0.0)$ \\
\hline
\end{tabular}

The COVID-19 Pandemic on GI Endoscopy
Digestion 2022;103:7-21

DOI: $10.1159 / 000520287$ 
Table 1 (continued)

\begin{tabular}{|c|c|c|c|c|c|c|c|c|}
\hline \multicolumn{9}{|c|}{ PPE in short supply after the first wave, $n(\%)$} \\
\hline None & $17(28.3)$ & $19(73.1)$ & $4(26.7)$ & $3(42.9)$ & $14(60.9)$ & $4(50.0)$ & $6(33.3)$ & $9(100.0)$ \\
\hline Goggle & $16(26.7)$ & $2(7.7)$ & $1(6.7)$ & $1(14.3)$ & $1(4.3)$ & $2(25.0)$ & $6(33.3)$ & $0(0.0)$ \\
\hline Surgical mask & $26(43.3)$ & $4(15.4)$ & $3(20.0)$ & $2(28.6)$ & $2(8.7)$ & $3(37.5)$ & $10(55.6)$ & $0(0.0)$ \\
\hline N95, FFP 2, or DS2 & $24(40.0)$ & $7(26.9)$ & $10(66.7)$ & $4(57.1)$ & $9(39.1)$ & $1(12.5)$ & $12(66.7)$ & $0(0.0)$ \\
\hline Hairnet & $14(23.3)$ & $1(3.8)$ & $0(0.0)$ & $1(14.3)$ & $0(0.0)$ & $2(25.0)$ & $9(50.0)$ & $0(0.0)$ \\
\hline Disposable gown & $32(53.3)$ & $3(11.5)$ & $7(46.7)$ & $3(42.9)$ & $4(17.4)$ & $3(37.5)$ & $10(55.6)$ & $0(0.0)$ \\
\hline Shoe covers & $8(13.3)$ & $1(3.8)$ & $0(0.0)$ & $2(28.6)$ & $1(4.3)$ & $1(12.5)$ & $9(50.0)$ & $0(0.0)$ \\
\hline \multicolumn{9}{|c|}{ PPE used during endoscopic procedure for low-risk patients, $n(\%)$} \\
\hline None & $1(1.7)$ & $3(11.5)$ & $0(0.0)$ & $2(28.6)$ & $0(0.0)$ & $0(0.0)$ & $0(0.0)$ & $0(0.0)$ \\
\hline Face shield & $37(61.7)$ & $11(42.3)$ & $11(73.3)$ & $3(42.9)$ & $23(100.0)$ & $6(75.0)$ & $16(88.9)$ & 8 (88.9) \\
\hline Hairnet & $32(53.3)$ & $11(42.3)$ & $7(46.7)$ & $0(0.0)$ & $22(95.7)$ & $4(50.0)$ & $14(77.8)$ & 7 (77.8) \\
\hline Disposable gown & $57(95.0)$ & $13(50.0)$ & $15(100.0)$ & $4(57.1)$ & $22(95.7)$ & $7(87.5)$ & $14(77.8)$ & $9(100.0)$ \\
\hline Shoe covers & $8(13.3)$ & $13(50.0)$ & $1(6.7)$ & $1(14.3)$ & $18(78.3)$ & $3(37.5)$ & $10(55.6)$ & $2(22.2)$ \\
\hline \multicolumn{9}{|c|}{ PPE using during endoscopic procedures for high-risk patients, $n(\%)$} \\
\hline None & $2(3.3)$ & $0(0.0)$ & $0(0.0)$ & $0(0.0)$ & $0(0.0)$ & $0(0.0)$ & $0(0.0)$ & $0(0.0)$ \\
\hline Face shield & $42(70.0)$ & $19(73.1)$ & $15(100.0)$ & $6(85.7)$ & $22(95.7)$ & $8(100.0)$ & $17(94.4)$ & $8(88.9)$ \\
\hline Goggle & $41(68.3)$ & $23(88.5)$ & $10(66.7)$ & $3(42.9)$ & $20(87.0)$ & $7(87.5)$ & $12(66.7)$ & $5(55.6)$ \\
\hline Surgical mask & $30(50.0)$ & $13(50.0)$ & $3(20.0)$ & $2(28.6)$ & $14(60.9)$ & $4(50.0)$ & $14(77.8)$ & $0(0.0)$ \\
\hline N95, FFP 2, or DS2 & $43(71.7)$ & $25(96.2)$ & $15(100.0)$ & $6(85.7)$ & $22(95.7)$ & 7 (87.5) & $18(100.0)$ & $9(100.0)$ \\
\hline Disposable gloves & $51(85.0)$ & $24(92.3)$ & $14(93.3)$ & $7(100.0)$ & $23(100.0)$ & 7 (87.5) & $16(88.9)$ & $9(100.0)$ \\
\hline Hairnet & $46(76.7)$ & $19(73.1)$ & $9(60.0)$ & $2(28.6)$ & $22(95.7)$ & 7 (87.5) & $16(88.9)$ & $6(66.7)$ \\
\hline Disposable gown & $50(83.3)$ & $23(88.5)$ & $15(100.0)$ & $6(85.7)$ & $22(95.7)$ & 7 (87.5) & $16(88.9)$ & $9(100.0)$ \\
\hline
\end{tabular}

JPN, Japan; CHN, China; HKG, Hong Kong; KOR, South Korea; PHL, Philippines; THA, Thailand; IDN, Indonesia; SGP, Singapore; FFP, filtering face piece; DS, disposable solid; PAPR, powered air-purifying respirator; PPE, personal protective equipment; GI, gastrointestinal.

strictions was only 9 (5.4\%) during the first wave, it increased to 74 (44.6\%) after the first wave (Fig. 2).

Most patients were screened for SARS-CoV-2 infection prior to screening or follow-up endoscopies, therapeutic endoscopies, and urgent endoscopies with symptoms (148 [89.2\%], 143 [86.1\%], and 129 [77.7\%], respectively) and body temperature (147 [88.6\%], 143 [86.1\%], and 132 [79.5\%], respectively). The SARS-CoV-2 polymerase chain reaction test was performed prior to screening or follow-up endoscopies, therapeutic endoscopies, and urgent endoscopies in $67(40.4 \%), 83(50.0 \%)$, and 50 (30.1\%) of institutions, respectively (Fig. 3a-c). Regarding the number of medical workers in the endoscopic units, the number of doctors working in the endoscopic units was 1-3 in the highest number of respondents (56 [33.7\%]) during the first wave, and it increased to 4-6 in the highest number of respondents (42 [25.3\%]) after the first wave (Fig. $3 \mathrm{~d}$ ). The number of the other medical workers did not change significantly during and after the pandemic (Fig. 3e-g). Experience of performing endoscopies for patients with a definite diagnosis of COVID-19 was found in 31 respondents (18.7\%), and COVID-19 infection from patients to medical workers through GI endoscopy was confirmed in 3 respondents (1.8\%). In contrast, COVID-19 infection from medical workers to patients through GI endoscopy was not confirmed (Fig. 3h, i). The data are listed in online supplementary Table 3. 


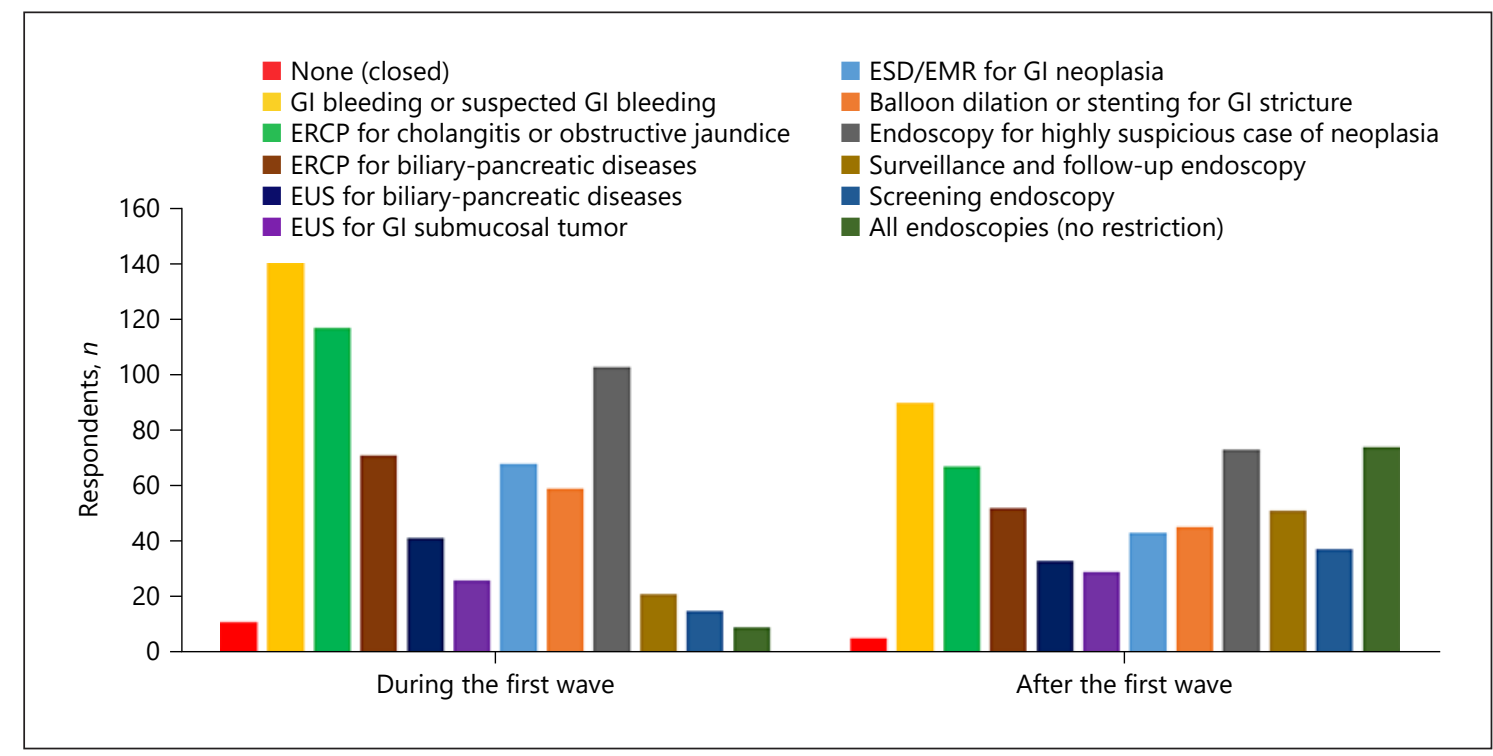

Fig. 2. The indications for GI endoscopy during and after the first wave. ERCP, endoscopic retrograde cholangiopancreatography; EUS, endoscopic ultrasound; ESD, endoscopic submucosal dissection; EMR, endoscopic mucosal resection; GI, gastrointestinal.

\section{GI Symptoms with COVID-19 and GI Diseases}

Affected by the Pandemic

It was found that 40 respondents (24.1\%) had experience of treating COVID-19 patients (Fig. 4a). The common frequency of GI symptoms in COVID-19 patients was $1-10 \%$ (15 [37.5\%]) or 11-25\% (13 [32.5\%]) (Fig. 4b). The most common GI symptom of COVID-19 patients during infected period was diarrhea (22 [55.0\%]), followed by nausea (18 [45.0\%]), and vomiting (9 [22.5\%]) (Fig. 4c). These GI symptoms most often appeared at the same time as respiratory symptoms (19 [47.5\%]) (Fig. 4d).

Some GI diseases, such as functional dyspepsia (73 [44.0\%]) and irritable bowel syndrome (61 [36.7\%]), were exacerbated by the COVID-19 pandemic (Fig. 4e). It should be noted that 65 respondents $(39.2 \%)$ reported cases with delayed diagnosis of cancers due to postponed endoscopic procedures (Fig. 4f). The most common type of cancer with a delayed diagnosis was colorectal cancer (59 [35.5\%]), followed by gastric cancer (46 [27.7\%]), esophageal cancer (28 [16.9\%]), small intestinal cancer $(10[6.0 \%])$, and duodenal cancer $(9$ [5.4\%]). In addition to these GI cancers, pancreatic cancer $(13$ [7.8\%]), cholangiocarcinoma (8 [4.8\%]), and hepatocellular carcinoma (1 [0.6\%]) were also delayed to be diagnosed (Fig. 4g). Furthermore, the new, continued, and resumed prescriptions of proton pump inhibitors/potassium-competitive acid blockers, steroids, immunosuppressive agents, and biologics, but not anticancer agents, were delayed or canceled due to the pandemic (Fig. 4h). The data are listed in online supplementary Table 4.

\section{Usage of PPE during Endoscopic Procedures}

The most deficient PPE during and after the first wave of the COVID-19 pandemic were N95 or equivalent masks (116 [69.9\%] and 67 [40.4\%], respectively), disposable gowns (106 [63.9\%] and 62 [37.3\%], respectively), and surgical masks (97 [58.4\%] and 50 [30.1\%], respectively) (Fig. 5a). There was no serious lack of PPE in Singapore, with 7 respondents $(77.8 \%)$ during the first wave and 9 respondents (100.0\%) after the first wave who answered none. In contrast, there was a serious shortage of surgical masks and N95 or equivalent masks (8 [100.0\%] and 7 [87.5\%], respectively) during the first wave in Thailand (Table 1). Commonly used PPE during endoscopic procedures for low-risk patients were disposable gloves (147 [88.6\%]), disposable gowns (141 [84.9\%]), surgical masks (116 [69.9\%]), and face shields (115 [69.3\%]), while those for high-risk patients were disposable gloves (151 [91.0\%]), disposable gowns (148 [89.2\%]), N95 or equivalent masks (145 [87.3\%]), and face shields (137 [82.5\%]) (Fig. 5b). N95 or equivalent masks were used almost $100 \%$ for low-risk patients during endoscopic procedures in Philippines, Indonesia, and Singapore, while they were more likely to be used for high-risk patients in other 


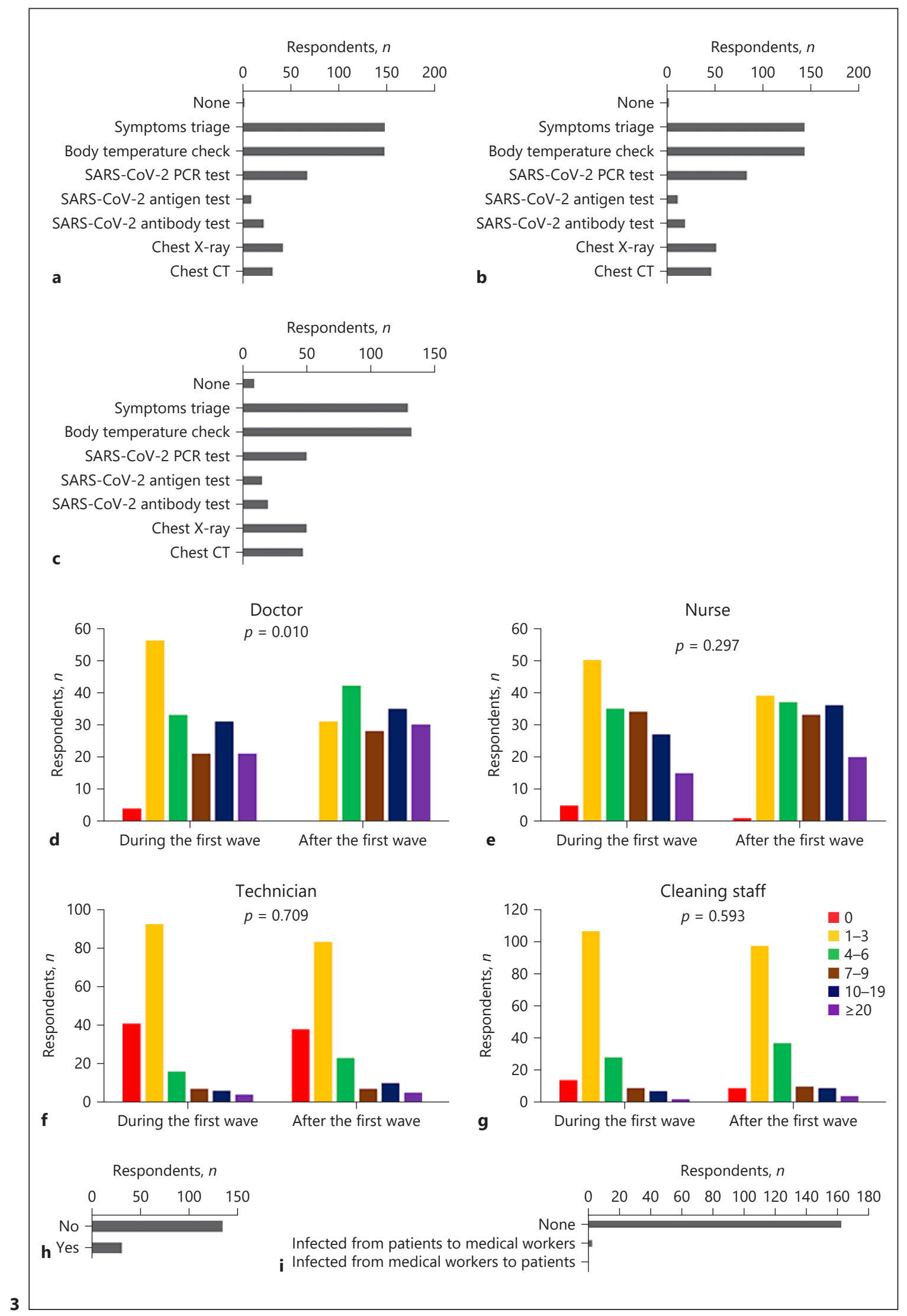

(For legend see next page.)

Otani et al. 
countries. For high-risk patients, medical protecting coverall was used by 1 respondent (12.5\%) in Thailand, hazmat suits were used by 2 respondents $(8.7 \%)$ in the Philippines, and a powered air-purifying respirator (PAPR) was used by 5 respondents in total in the Philippines and Singapore (Table 1). To prevent the diffusion of aerosol droplets, the major devices used during endoscopic procedures were modified surgical masks for examinees (62 [37.3\%]), disposable vinyl boxes covering the head of patients (23 [13.9\%]), mouthpieces with filters (19 [11.4\%]), and aerosol boxes covering the head of patients $(17[10.2 \%])$. It was found that 72 respondents (43.4\%) did not use devices on the patient side during the endoscopic procedures (Fig. 5c).

The level of PPE during the endoscopic procedure was considered appropriate by 145 respondents $(86.8 \%)$ and inadequate by 19 respondents (11.4\%) (Fig. 5d). The PPE considered the most excessive was N95 or equivalent masks (3 [33.3\%]) (Fig. 5e). On the other hand, the PPE considered inadequate were N95 or equivalent masks (5 [23.8\%]) and aerosol boxes covering the head of patients (3 [14.3\%]) (Fig. 5f). A total of 113 respondents (68.1\%) answered that the present level of PPE should be maintained during future endoscopic procedures, even after the pandemic. However, 53 respondents (31.9\%) answered that they would discontinue the use of present PPE after the pandemic, and the most common reason was high cost (37 [69.8\%]) (Fig. 5g, h). The data are listed in online supplementary Table 5.

\section{Impact of the COVID-19 Pandemic on Education, \\ Research, and Clinical Practice}

It was revealed that the time for education, basic research, clinical research, and daily clinical practice decreased during the first wave, compared to before the pandemic. It significantly recovered after the first wave,

Fig. 3. The way to assess the risk of SARS-CoV-2 infection before GI endoscopies and impact of the COVID-19 pandemic on the number of medical staff. a The way to assess the risk of SARSCoV-2 infection before screening or follow-up endoscopies. $\mathbf{b}$ The way to assess the risk of SARS-CoV-2 infection before therapeutic endoscopies. $c$ The way to assess the risk of SARS-CoV-2 infection before urgent endoscopies (on call). $\mathbf{d}-\mathbf{g}$ The number of medical staff working in the endoscopic units during and after the first wave. $\mathbf{d}$ Doctor. e Nurse. $\mathbf{f}$ Technician. $\mathbf{g}$ Cleaning staff. $\mathbf{h}$ The experience of performing endoscopy for patients with a definite diagnosis of COVID-19. i COVID-19 case obviously infected through GI endoscopy. PCR, polymerase chain reaction; CT, computed tomography; SARS-CoV-2, severe acute respiratory syndrome coronavirus 2; GI, gastrointestinal.

The COVID-19 Pandemic on GI Endoscopy but not completely (Fig. 6a-d). The data are listed in online supplementary Table 6 .

\section{Discussion}

This is a questionnaire-based survey investigating the effects of the first wave of the COVID-19 pandemic on GI endoscopy practice across multiple facilities in East Asia and Southeast Asia. Although it was known that the practice of GI endoscopy was restricted during the first wave of the pandemic, the quantitative evaluation of impact of the pandemic on the number of GI endoscopies and medical staff, GI diseases, PPE supply, and medical activities has been inadequate. As most of the respondents were staff or directors who specialize in gastroenterology, we consider that this study reflects the views of Asian GI experts on the current situation of the COVID-19 pandemic.

The volume of screening or follow-up endoscopies and therapeutic endoscopies was markedly reduced during the first wave of the pandemic because it was strongly recommended that the nonurgent endoscopies should be postponed or canceled to prevent the spread of SARSCoV-2 by academic societies on endoscopy, and most doctors focused on and valued the statements. The reduction was also due to decreased number of outpatients and cancellations by patients, suggesting that patients were highly aware of the risk of SARS-CoV-2 infection by endoscopy and visiting hospitals. Furthermore, not a few respondents raised the limited number of medical workers in the endoscopic units as a reason for the decreased endoscopic volume. Therefore, we should know the changes in the number of medical workers working in the endoscopic units during the first wave. Our survey showed that the number of doctors working in endoscopic units decreased during the first wave, suggesting that the doctors diverted from GI endoscopy services to the other medical areas in greater need during the first wave. After the first wave, the volume of screening or follow-up endoscopies and therapeutic endoscopies did not return completely in most of Asian countries. However, it resumed considerably in Thailand, which might be because that the number of COVID-19 patients was completely controlled once by locking down in Thailand from the end of March 2020 and recommended PPE for a confirmed case with COVID-19 by the Thai Association for Gastrointestinal Endoscopy was strict [15]. It is recommended that the full capacity of endoscopic units should be resumed when there are no new cases of COVID-19 

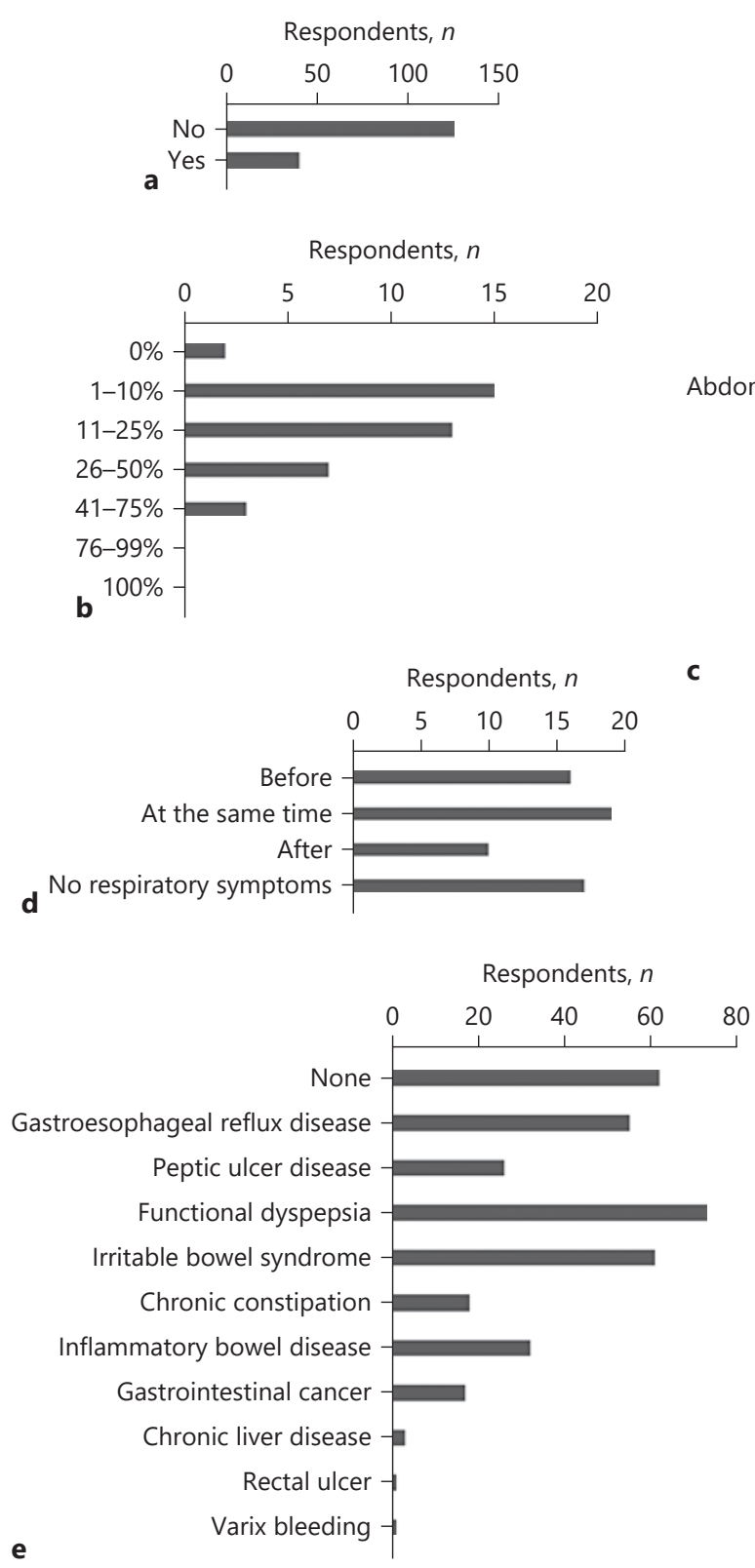

g

Abdominal bloating/distension

Vomiting

Epigastric pain

Abdominal pain

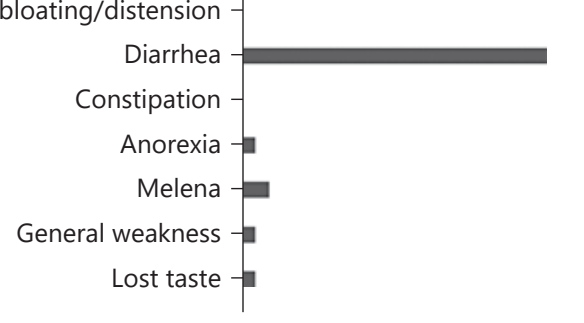

C
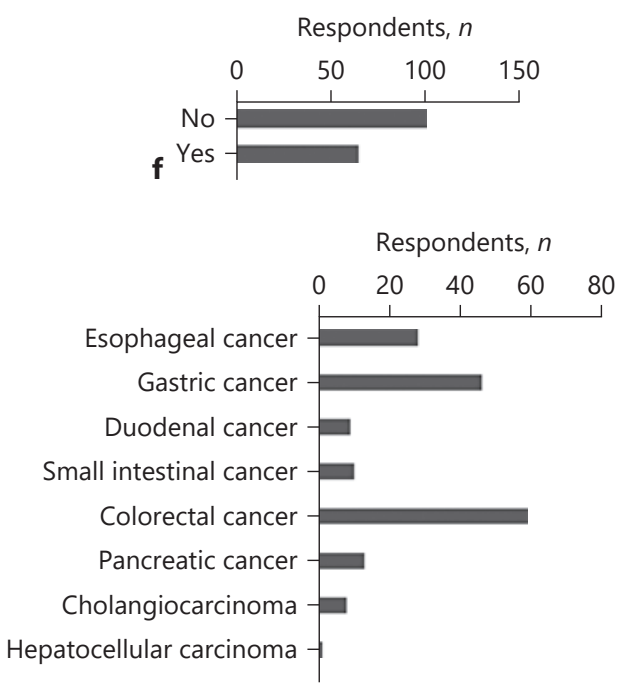

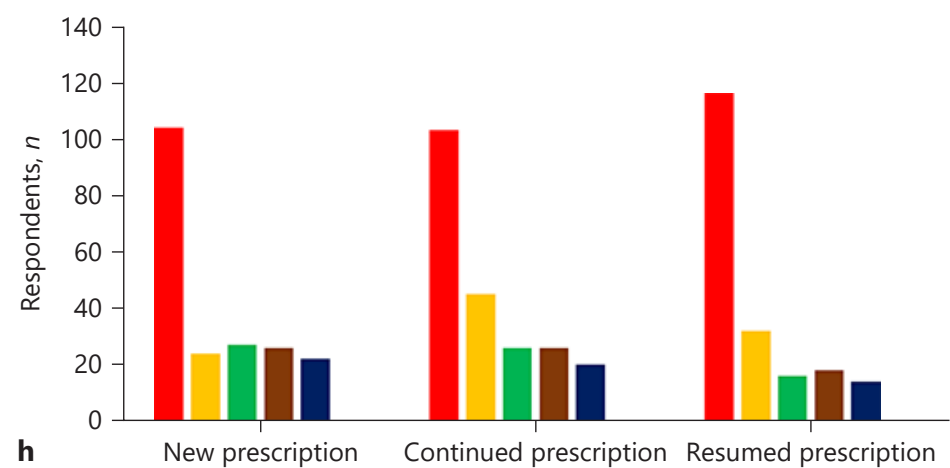

4

(For legend see next page.)

None

PPI/P-CAB

Steroid

Immunosuppressive agent

Biologics

Anti-cancer agent 
diagnosed for at least 2 weeks in APSDE-COVID statements [11]. In contrast, the decrease in the volume of urgent endoscopies was relatively mild, and priority was given to the examinations that are directly associated with patient life, such as those for GI bleeding, cholangitis or obstructive jaundice, and highly suspicious cases of neoplasia as expected. We found that the volume of urgent endoscopies did not decrease much during and after the first wave, particularly in Hong Kong. It is recommended that urgent endoscopies should be performed by strategically assigned staff to minimize concomitant exposure and the indications are limited to acute GI bleeding, management of perforations and leakage, biliary sepsis, foreign body, GI obstruction requiring stenting, and GI access for urgent feeding in APSDE-COVID statements [11].

In most institutions, symptom triage and body temperature check were performed before screening or follow-up endoscopies, therapeutic endoscopies, and urgent endoscopies. Although these seem to be the most appropriate methods to prevent the infection in extremely busy endoscopic units if the prevention with PPE is sufficient, 3 cases of SARS-CoV-2 infection from patients to medical workers were confirmed. This means that GI endoscopies are undoubtedly high-risk procedures for the transmission of SARS-CoV-2 [16]. There is a limit to assess the risk for SARS-CoV-2 before GI endoscopy because approximately $5 \%$ of COVID-19 patients were reported to be asymptomatic during the incubation period and could act as a source of infection [17].

The most common frequency of GI symptoms in COVID-19 patients was 1-25\%, and they were associated with acute viral gastroenteritis, such as diarrhea, nausea, and vomiting, which was almost consistent with previous reports $[18,19]$. The COVID-19 pandemic had a considerable impact not only on the practice of GI endoscopy

Fig. 4. GI symptoms with COVID-19 and GI diseases affected by the pandemic. a The experience of treating COVID-19 patients. $\mathbf{b}$ The frequency of GI symptoms in COVID-19 patients. c The GI symptoms of COVID-19 patients during the infected period (most common one). d The time that GI symptoms appeared (Before, before respiratory symptoms; At the same time, at the same time with respiratory symptoms; After, after respiratory symptoms). e The GI diseases exacerbated by the COVID-19 pandemic. $f$ The delayed diagnosis of cancers due to postponed endoscopic procedure. $\mathbf{g}$ The types of cancer with a delayed diagnosis due to postponed endoscopic procedure. $\mathbf{h}$ The prescription delayed or canceled due to the COVID-19 pandemic. PPI, proton pump inhibitor; $\mathrm{P}-\mathrm{CAB}$, potassium-competitive acid blocker; GI, gastrointestinal.

The COVID-19 Pandemic on GI Endoscopy but also on the medical treatment for GI diseases. Among GI diseases, it was found that functional dyspepsia and irritable bowel syndrome were exacerbated by the COVID-19 pandemic. The COVID-19 pandemic is definitely a stressful situation for general population and functional dyspepsia and irritable bowel syndrome are well known to be aggravated by psychosocial stress [20,21]. Alarmingly, it was found that postponed endoscopy delayed the diagnosis of GI cancers, such as colorectal cancer and gastric cancer. As most patients with early GI cancers are asymptomatic, triaging patients at high risk of developing cancer may have been difficult. It was reported that the endoscopic detection for GI cancers was reduced by $58 \%$ during the COVID-19-impacted period in a study using a national endoscopy database [13]. Therefore, the postponement of endoscopy should be minimized, and attention should be paid to patients at high risk of developing cancer. Furthermore, it was clarified that the new, continued, and resumed prescriptions of acid suppressants, steroids, immunosuppressive agents, and biologics were delayed by the pandemic. It has been reported that the administration of proton pump inhibitor increases the risk of developing infectious diseases, such as pneumonia and infectious enteritis [22, 23]. Moreover, it is recommended that the dose of corticosteroids should be tapered whenever possible after assessing the effects on patients with inflammatory bowel disease and immunosuppressive agents and biologics should be discontinued or postponed for patients with inflammatory bowel disease in contact with COVID-19 patients as they are considered potential risk factors [24, 25]. However, there were no respondents who delayed or canceled the prescription of anticancer agents, probably to adhere to the dosing schedule of chemotherapy. Despite the lack of evidence regarding the risk of anticancer agents for COVID-19, doctors should be aware that lung injury induced by molecular-targeted agents and immune checkpoint inhibitors may be indistinguishable from COVID-19 pneumonia [26, 27].

Shortage of PPE is a severe global issue, particularly in the early days of the COVID-19 pandemic [28]. Most respondents in this survey reported that they experienced a serious PPE shortage, such as N95 or equivalent masks, disposable gowns, and surgical masks during the first wave. The shortage gradually improved. However, more than half of the respondents, except those in Singapore, reported that they continued to face some shortages after the first wave. It has been reported that the common PPE used for patients with COVID-19 during endoscopic procedures included surgical masks and N95 masks, face 


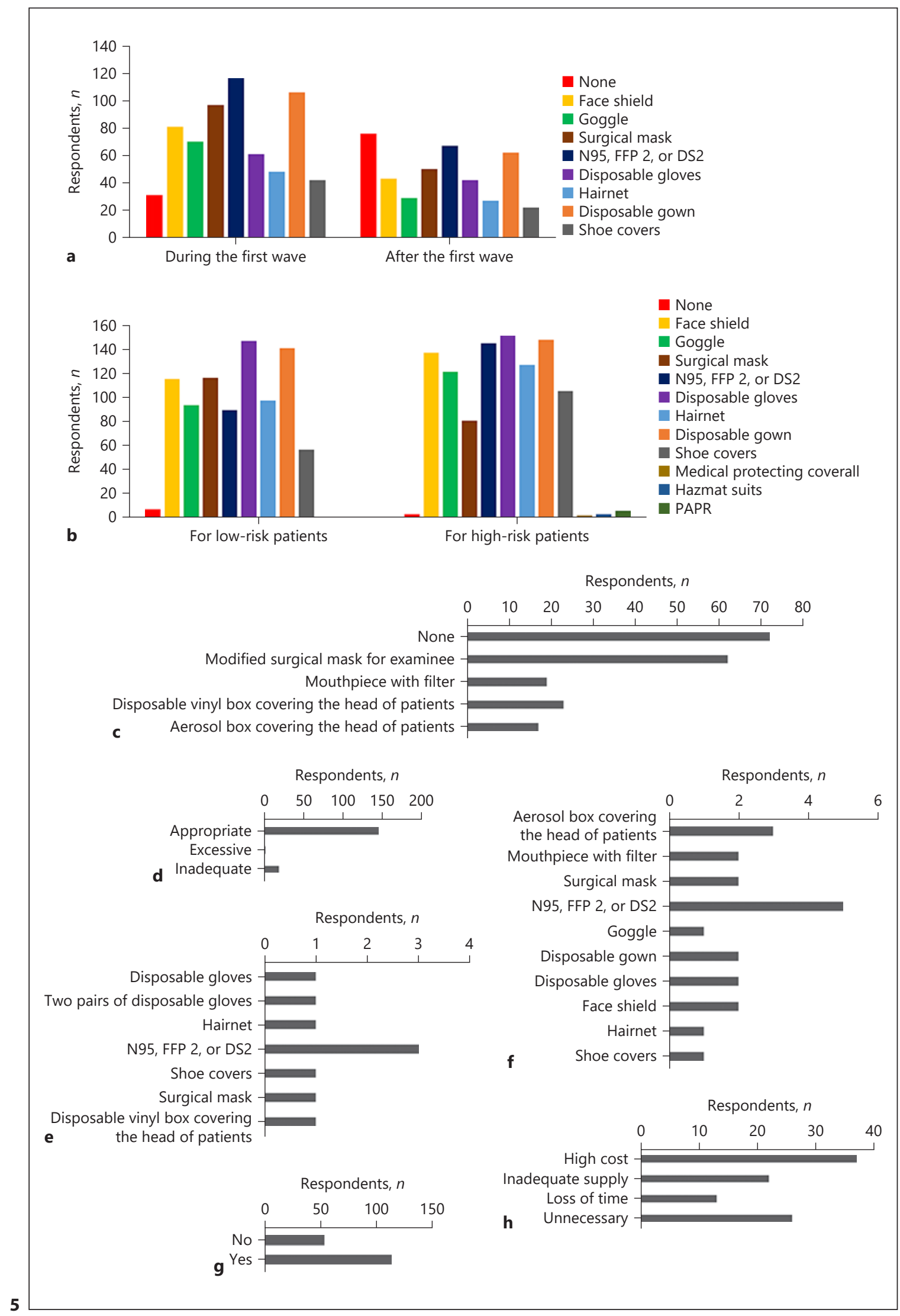

(For legend see next page.)

Otani et al. 
shields, goggles, caps, long-sleeved isolation gowns, and gloves [29], which are almost consistent regarding the results of PPE for high-risk patients in our study. N95 or equivalent masks were used more frequently for high-risk patients than for low-risk patients. Medical protecting coverall or hazmat suits were used by a few respondents for high-risk patients in the Philippines and Thailand. The endoscopist is recommended to wear medical protecting coverall for a confirmed case with COVID-19 by Thai Association for Gastrointestinal Endoscopy [15]. Notably, PAPR was used by a few respondents for highrisk patients in the Philippines and Singapore. The use of PAPR for GI endoscopic procedures in high-risk patients is recommended by gastroenterologists' professional guidance on risk mitigation during the COVID-19 pan- demic in Singapore [30]. As devices on the patient side, modified surgical masks, mouthpieces with filters, and disposable vinyl boxes or aerosol boxes covering the head of patients were relatively well used as previously reported [31-33]. As most respondents considered that the current required level of PPE was appropriate and should be maintained during endoscopy, the statements on PPE of academic societies were appropriate and should be adhered to for the time being to protect medical workers and patients.

We finally investigated the impact of the COVID-19 pandemic on education, basic research, clinical research, and daily clinical practice. In this survey, approximately $50.0 \%$ of respondents answered that the activities of education, basic research, and clinical research decreased

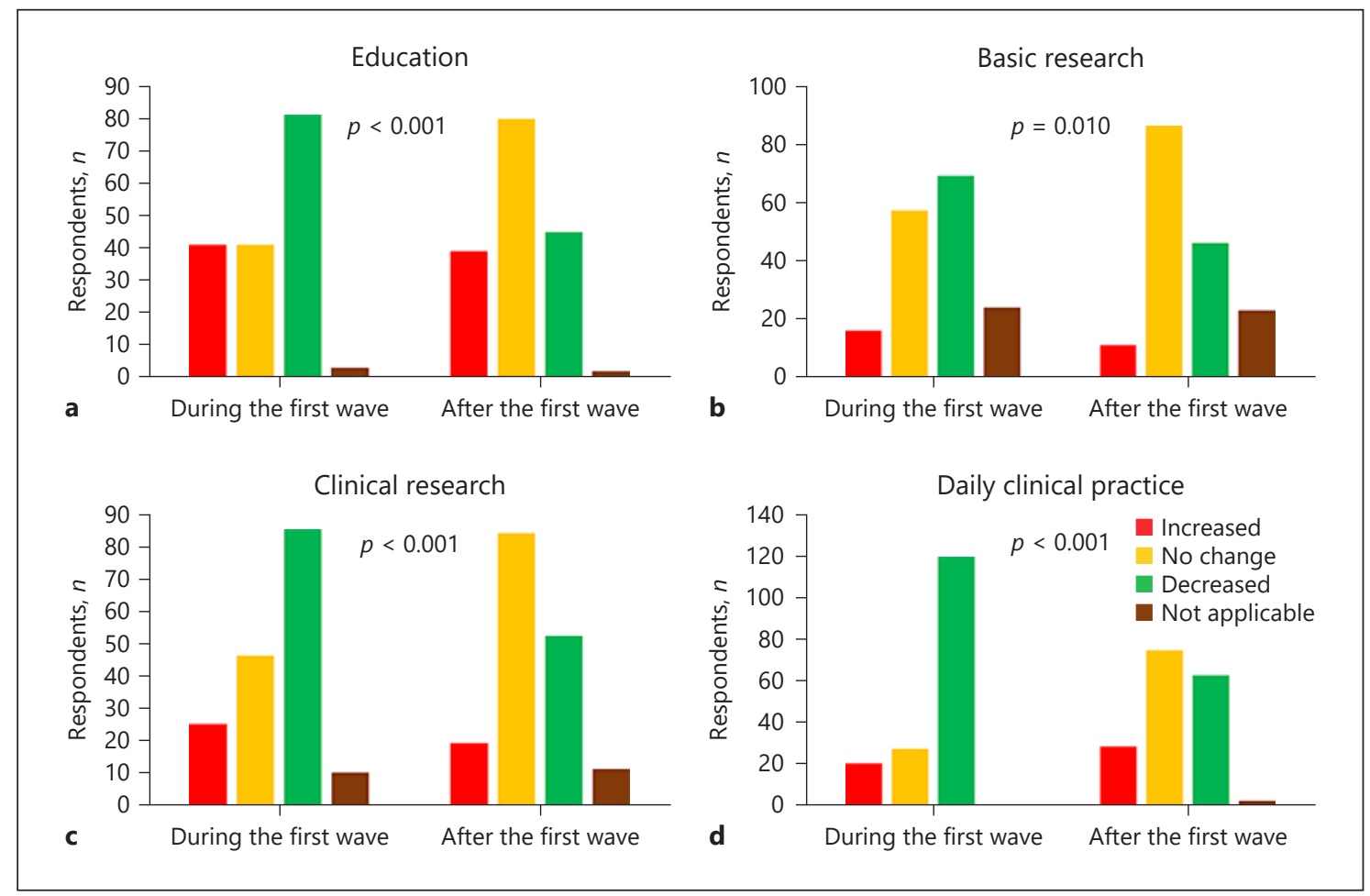

Fig. 6. Impact of the COVID-19 pandemic on education, research, and clinical practice. a Time of education affected by the COVID-19 pandemic compared to before the pandemic. b Time of basic research affected by the COVID-19 pandemic compared to before the pandemic. c Time of clinical research affected by the COVID-19 pandemic compared to before the pandemic. d Time of daily clinical practice affected by the COVID-19 pandemic compared to before the pandemic.
Fig. 5. Usage of PPE during endoscopic procedures. a PPE in short supply. b PPE used during endoscopic procedures. c The devices for preventing diffusion of aerosol droplets used during the endoscopic procedure. d The level of PPE during endoscopic procedures. e The excessive PPE. $\mathbf{f}$ The inadequate PPE. g Maintaining the present level of PPE during the endoscopic procedure after the COVID-19 pandemic. $\mathbf{h}$ The reasons for discontinuation of the present level of PPE after the COVID-19 pandemic. FFP, filtering face piece; DS, disposable solid; PAPR, powered air-purifying respirator; PPE, personal protective equipment. 
during the first wave. Although many classes were conducted online using a video conferencing system, medical students missed the opportunity to learn clinical skills competence due to the suspension of clinical placements [34]. Residents and fellows also suffered from the disadvantage of losing the opportunity for endoscopic training due to restrictions in endoscopic volume, with high rates of anxiety and burnout worldwide [35]. Moreover, basic research and clinical research were limited to be carried out, which may be due to maintaining social distance and prohibiting people from entering the institution. Daily clinical practice was particularly restricted during the first wave, which may be attributable to avoiding the spread of SARS-CoV-2 infection through endoscopies and outpatients and keeping the beds for COVID-19 patients instead of general inpatients.

This study has several limitations. First, the timing of the first wave, the degree of lockdown or restriction, the number of serious cases with COVID-19, and the guidelines for using PPE during GI endoscopy differed depending on each city and country. Second, this was a retrospective observational study after the first wave, and changes in the situation over time cannot be observed. Third, there is a possibility that there are more cases with delayed diagnosis of cancers due to postponed endoscopy because the cancers could not be diagnosed until the rescheduled endoscopy at a later date. As the COVID-19 situation is changing rapidly across the world, it is necessary to continue objective research to examine accurate medical conditions.

\section{Conclusion}

In conclusion, this study demonstrated that the COVID-19 pandemic profoundly affected the method of GI endoscopy and medical treatment for patients with GI diseases in Asian countries. We hope that this study will be helpful for the practice of GI endoscopy and GI diseases during a future outbreak of the COVID-19 pandemic.

\section{Acknowledgments}

The authors thank all doctors who participated in this survey and also the office personnel of the IGICS for their secretarial work.

\section{Statement of Ethics}

This survey is questionnaire based not to patients but to each institution, and personal information is not handled. This kind of study does not require the approval of ethical review board according to the Japanese "guidelines for medical and health research involving human subjects" by Japanese Ministry of Health, Labor, and Welfare. Written informed consent of the participants was inferred from survey completion.

\section{Conflict of Interest Statement}

The authors declare no conflicts of interest.

\section{Funding Sources}

The authors have no funding sources.

\section{Author Contributions}

K.O. developed the questionnaire, analyzed the data, and drafted the paper; T.W. supervised the study and revised the paper; A.H. developed the questionnaire; and H.S., T.K., A.S., M.S., A.N., S.F., S.M., S.Y., Q.Z., F.K.L.C., K.-B.H., M.C.T., V.P., M.A., T.L.A., and K.M. cooperated with this study and provided valid inputs.

\section{Data Availability Statement}

All data analyzed in this study are included in this article. Further enquiries can be directed to the corresponding author.

\section{References}

1 Wang C, Horby PW, Hayden FG, Gao GF. A novel coronavirus outbreak of global health concern. Lancet. 2020 Feb 15;395(10223): 470-3.

2 Nussbaumer-Streit B, Mayr V, Dobrescu AI, Chapman A, Persad E, Klerings I, et al. Quarantine alone or in combination with other public health measures to control COVID-19: a rapid review. Cochrane Database Syst Rev. 2020 Sep 15;4:CD013574.
3 Rice K, Wynne B, Martin V, Ackland GJ. Effect of school closures on mortality from coronavirus disease 2019: old and new predictions. BMJ. 2020 Oct 7;371:m3588.

4 Woskie LR, Hennessy J, Espinosa V, Tsai TC, Vispute S, Jacobson BH, et al. Early social distancing policies in Europe, changes in mobility \& COVID-19 case trajectories: insights from Spring 2020. PLoS One. 2021;16(6): e0253071. 
5 Han Q, Lin Q, Ni Z, You L. Uncertainties about the transmission routes of 2019 novel coronavirus. Influenza Other Respir Viruses. 2020 Jul;14(4):470-1.

6 Tian Y, Rong L, Nian W, He Y. Review article: gastrointestinal features in COVID-19 and the possibility of faecal transmission. Aliment Pharmacol Ther. 2020 May;51(9):843-51.

7 Wang J, Du G. COVID-19 may transmit through aerosol. Ir J Med Sci. 2020 Nov; 189(4):1143-4.

8 Wang W, Xu Y, Gao R, Lu R, Han K, Wu G, et al. Detection of SARS-CoV-2 in different types of clinical specimens. JAMA. 2020 May 12;323(18):1843-4.

9 Wang Z, Fu Y, Guo Z, Li J, Li J, Cheng H, et al. Transmission and prevention of SARSCoV-2. Biochem Soc Trans. 2020 Oct 30; 48(5):2307-16

10 Hussain A, Singhal T, El-Hasani S. Extent of infectious SARS-CoV-2 aerosolisation as a result of oesophagogastroduodenoscopy or colonoscopy. Br J Hosp Med. 2020 Jul 2;81(7): $1-7$.

11 Chiu PWY, Ng SC, Inoue H, Reddy DN, Ling $\mathrm{Hu}$ E, Cho JY, et al. Practice of endoscopy during COVID-19 pandemic: position statements of the Asian Pacific Society for digestive endoscopy (APSDE-COVID statements). Gut. 2020 Jun;69(6):991-6.

12 Irisawa A, Furuta T, Matsumoto T, Kawai T, Inaba T, Kanno A, et al. Gastrointestinal endoscopy in the era of the acute pandemic of coronavirus disease 2019: recommendations by Japan Gastroenterological Endoscopy Society (issued on April 9th, 2020). Dig Endosc. 2020 Jul;32(5):648-50.

13 Rutter MD, Brookes M, Lee TJ, Rogers P, Sharp L. Impact of the COVID-19 pandemic on UK endoscopic activity and cancer detection: a National Endoscopy Database Analysis. Gut. 2021 Mar;70(3):537-43.

14 Boskoski I, Gallo C, Wallace MB, Costamagna G. COVID-19 pandemic and personal protective equipment shortage: protective efficacy comparing masks and scientific methods for respirator reuse. Gastrointest Endosc. 2020 Sep;92(3):519-23.

15 Kongkam P, Tiankanon K, Ratanalert S, Janthakun V, Kitiyakara T, Angsuwatcharakon $\mathrm{P}$, et al. The practice of endoscopy during the COVID-19 pandemic: recommendations from the Thai Association for gastrointestinal endoscopy (TAGE) in collaboration with the Endoscopy Nurse Society (Thailand). Siriraj Med J. 2020;72(4):283-6.
16 Crespo J, Iglesias-García J, Hinojosa Del Val JE, García García F, Gil de Miguel Á, Fernández Carrillo C, et al. COVID-19 and the digestive system: protection and management during the SARS-CoV-2 pandemic. Rev Esp Enferm Dig. 2020 May;112(5):389-96.

17 Zhu J, Zhong Z, Ji P, Li H, Li B, Pang J, et al. Clinicopathological characteristics of 8697 patients with COVID-19 in China: a metaanalysis. Fam Med Community Health. 2020 Apr;8(2):e000406.

18 Lin L, Jiang X, Zhang Z, Huang S, Zhang Z, Fang Z, et al. Gastrointestinal symptoms of 95 cases with SARS-CoV-2 infection. Gut. 2020 Jun;69(6):997-1001.

19 Redd WD, Zhou JC, Hathorn KE, McCarty TR, Bazarbashi AN, Thompson CC, et al. Prevalence and characteristics of gastrointestinal symptoms in patients with severe acute respiratory syndrome coronavirus 2 infection in the United States: a Multicenter Cohort Study. Gastroenterology. 2020 Aug;159(2): 765-7.e2.

20 Miwa $\mathrm{H}$ Life style in persons with functional gastrointestinal disorders - large-scale internet survey of lifestyle in Japan. Neurogastroenterol Motil. 2012 May;24(5):464-71.e217.

21 Fukudo S, Okumura T, Inamori M, Okuyama Y, Kanazawa M, Kamiya T, et al. Evidencebased clinical practice guidelines for irritable bowel syndrome 2020. J Gastroenterol. 2021 Mar;56(3):193-217.

22 Bavishi C, Dupont HL. Systematic review: the use of proton pump inhibitors and increased susceptibility to enteric infection. Aliment Pharmacol Ther. 2011 Dec;34(11-12):126981.

23 Lambert AA, Lam JO, Paik JJ, Ugarte-Gil C, Drummond MB, Crowell TA. Risk of community-acquired pneumonia with outpatient proton-pump inhibitor therapy: a systematic review and meta-analysis. PLoS One. 2015; 10(6):e0128004.

24 Magro F, Abreu C, Rahier JF. The daily impact of COVID-19 in gastroenterology. United European Gastroenterol J. 2020 Jun;8(5): 520-7.

25 Nakase H, Matsumoto T, Matsuura M, Iijima H, Matsuoka K, Ohmiya N, et al. Expert opinions on the current therapeutic management of inflammatory bowel disease during the COVID-19 pandemic: Japan IBD COVID-19 taskforce, intractable diseases, the Health and Labor Sciences Research. Digestion. 2021; 102(5):814-22.
26 Kudoh S, Kato H, Nishiwaki Y, Fukuoka M, Nakata K, Ichinose Y, et al. Interstitial lung disease in Japanese patients with lung cancer: a cohort and nested case-control study. Am J Respir Crit Care Med. 2008 Jun 15;177(12): 1348-57.

27 Nishino M, Ramaiya NH, Awad MM, Sholl LM, Maattala JA, Taibi M, et al. PD-1 inhibitor-related pneumonitis in advanced cancer patients: radiographic patterns and clinical course. Clin Cancer Res. 2016 Dec 15;22(24): 6051-60.

28 Cohen J, Rodgers YVM. Contributing factors to personal protective equipment shortages during the COVID-19 pandemic. Prev Med. 2020 Dec;141:106263.

29 Niikura R, Fujishiro M, Nakai Y, Matsuda K, Kawahara T, Yamada A, et al. International observational survey of the effectiveness of personal protective equipment during endoscopic procedures performed in patients with COVID-19. Digestion. $2021 \mathrm{Feb}$ 16:1-9. Epub ahead of print.

30 Ang TL, Li JW, Vu CKF, Ho GH, Chang JPE, Chong $\mathrm{CH}$, et al. Chapter of gastroenterologists professional guidance on risk mitigation for gastrointestinal endoscopy during $\mathrm{CO}$ VID-19 pandemic in Singapore. Singapore Med J. 2020 Jul;61(7):345-9.

31 Kobara H, Nishiyama N, Masaki T. Shielding for patients using a single-use vinyl-box under continuous aerosol suction to minimize SARS-CoV-2 transmission during emergency endoscopy. Dig Endosc. 2020 Jul;32(5):e1145.

32 Maruyama $\mathrm{H}$, Higashimori A, Yamamoto $\mathrm{K}$, Nakata A, Ishikawa-Kakiya Y, Yamamura M, et al. Coronavirus disease outbreak: a simple infection prevention measure using a surgical mask during endoscopy. Endoscopy. 2020 Dec;52(12):E461-2.

33 Sagami R, Nishikiori H, Sato T, Murakami K. Endoscopic shield: barrier enclosure during the endoscopy to prevent aerosol droplets during the COVID-19 pandemic. VideoGIE. 2020 Oct;5(10):445-8.

34 Sani I, Hamza Y, Chedid Y, Amalendran J, Hamza N. Understanding the consequence of COVID-19 on undergraduate medical education: medical students' perspective. Ann Med Surg. 2020 Oct;58:117-9.

35 Pawlak KM, Kral J, Khan R, Amin S, Bilal M, Lui RN, et al. Impact of COVID-19 on endoscopy trainees: an international survey. Gastrointest Endosc. 2020 Oct;92(4):925-35. 